\title{
Enseñar ciencias con actividades secuenciadas a partir de los intereses de los estudiantes: Una mirada desde las percepciones y aprendizajes
}

\section{Teach sciences with activities sequenced from the interests of students: A look from the perceptions and learning}

DOI: $10.46932 /$ sfjdv2n5-050

Received in: Oct 1st, 2021

Accepted in: Dec 30th, 2021

\author{
María José Olguín Muñoz \\ Profesora de Biología y Ciencias \\ Universidad Central de Chile. \\ E-mail: mariajosehdm@gmail.com \\ Carlos Vanegas-Ortega \\ Doctor en Ciencias de la Educación \\ Universidad de Santiago de Chile. \\ E-mail: cmariov@gmail.com \\ Paulina Guzmán Sánchez \\ Profesor de Biología y Ciencias \\ Universidad Central de Chile. \\ E-mail: pauu.gsz@hotmail.com \\ Matías Donoso Saldaña \\ Profesor de Biología y Ciencias \\ Universidad Central de Chile. \\ E-mail: donosomatias3@gmail.com

\section{Rodolfo Durán} \\ Profesor de Biología y Ciencias \\ Universidad Central de Chile. \\ E-mail: rodoignacio94@gmail.com
}

\section{RESUMEN}

La organización y la secuenciación de contenidos y actividades de enseñanza son un punto neurálgico para la enseñanza de las ciencias, debido al tiempo, metodologías y otros conocimientos profesionales. El objetivo de esta investigación es explicar cómo influye la secuenciación de actividades a partir de los intereses de los estudiantes, en sus percepciones sobre la enseñanza de las ciencias y en los aprendizajes de nociones científicas específicas. La investigación es mixta y cuenta con tres fases para detectar intereses de los estudiantes. Los resultados muestran que los estudiantes lograron altos niveles de aprendizajes y sus percepciones mejoraron, llevándolos a ser más partícipes de las clases y a proponer mejoras generales, disposicionales y concretas para la enseñanza de las ciencias.

Palabras clave: percepciones sobre las ciencias, intereses de los estudiantes, secuenciación de actividades, conocimiento didáctico del contenido. 


\begin{abstract}
The organization and sequencing of content and teaching activities are a key point for the teaching of science, due to the time, methodologies and other professional knowledge. The objective of the research is to explain how the sequencing of activities influences the interests of students, their perceptions about the teaching of science and the learning of specific scientific notions. The research is mixed and has three phases to detect student interests. The results show that the students achieved high levels of learning and their perceptions improved, leading them to be more involved in the classes and to propose general improvements, dispositional and concrete ones for the teaching of sciences.
\end{abstract}

Key words: perceptions about the sciences, interests of the students, sequencing of activities, pedagogical content knowledge.

\title{
1 INTRODUCCIÓN
}

La organización y la secuenciación de los contenidos de enseñanza son un punto neurálgico para la enseñanza de las ciencias, debido al tiempo, metodologías y otros conocimientos profesionales que debe utilizar el profesorado. La selección de contenidos conlleva un proceso de toma de decisiones sobre la base de criterios explícitos que corresponden a la significación lógica, representatividad, actualidad, relevancia y contextualización de la enseñanza.

La literatura muestra que existe evidencia empírica para concluir que la secuenciación de contenidos debe ser un proceso de co-creación entre el profesor y los estudiantes (Adúriz-Bravo, Izquierdo \& Estany, 2002; Dewitt 1.1., 2013; Gunstone, 2012; Díaz-Jatuf, 2019; Izquierdo, 2005; Yang, Guo \& Yu, 2016; Zapata, 2005). Por ello, el objetivo de esta investigación es explicar cómo influye la secuenciación de actividades a partir de los intereses de los estudiantes, en sus percepciones sobre la enseñanza de las ciencias y en los aprendizajes de nociones científicas específicas.

Según Díaz-Jatuf (2019), la selección de contenido debe realizarse a través de las necesidades derivadas de los estudiantes, que generen un propósito de orientación de las acciones e intervenciones formativas que se realizan. La selección de contenidos debería: a) responder a las necesidades específicas de los alumnos en un determinado contexto; b) ser coherente con las opciones que confieren carácter propio a la institución que las organiza o al programa formativo, o al centro; c) estar dentro de un contexto socioeconómico y cultural y; d) incluir los elementos preceptivos del currículo escolar. Luego de realizar la selección de contenidos, los profesores se ven ante un desafío mayor: la secuenciación de los contenidos (Jack \& Lin, 2014; Penuel, Gallagher \& Moorthy, 2011; Wilson, 2013). La selección, organización y secuenciación de los contenidos, son desafío profesional para el profesorado porque exigen concreción en los objetivos educativos que orientará los procesos de enseñanza y de aprendizaje (Zapata, 2005). Es necesario señalar que los diversos componentes del currículo (fundamentos, objetivos, contenidos, evaluación y recursos), tienen como problema que habitualmente se tratan por separado, y estos deberían 
estar interrelacionados (Díaz-Jatuf, 2019; Izquierdo, 2005). De manera que, cuando sean abordados tanto desde la planificación como en la propia intervención formativa y en la evaluación, se tendría que ir revisando constantemente la práctica docente en función de los resultados obtenidos, comparándolos con los objetivos previstos y tomando decisiones sobre las mejoras pertinentes (Zapata, 2005).

Una investigación realizada con docentes de ciencias naturales de Colombia, mostró tres antecedentes relevantes sobre la secuenciación de actividades para la enseñanza de las ciencias (Daza, Arrieta \& Ríos, 2014): a) el 80\% de los profesores entrevistados seleccionan contenidos teniendo en cuenta los programas académicos, un $18 \%$ modificaba el orden del programa y un $2 \%$ introducían temas científicos de la actualidad. Dado esto, los conocimientos que pretenden enseñar los profesores se convierten en contenidos independientes y tienen el mismo nivel de complejidad, sin importar el orden y la distribución; b) los profesores siguen la lógica de la disciplina, el que proponen los libros de textos o el orden que siguen los científicos en la construcción de conocimientos a lo largo de la historia; c) lo que enseña el docente en las aulas difiere significativamente del currículo oficial.

Complementario a lo anterior, Sánchez Blanco y Valcárcel Pérez (2000) demostraron que la programación anual se realiza utilizando como elemento clave el contenido disciplinar o basándose en las planificaciones de años anteriores y la experiencia docente; lo cual, en muchos casos, representa simplificaciones progresivas de los contenidos científicos. Además, en ningún caso los profesores mencionan criterios científicos o didácticos para explicar los criterios de secuenciación de los contenidos.

Los docentes al momento de seleccionar y secuenciar los contenidos de enseñanza, pocas veces tienen en cuenta la edad cronológica de los estudiantes y, por tanto, el nivel cognitivo de ellos. Desde un punto de vista psicogenético, los niveles de desarrollo "permiten definir lo que un individuo puede comprender, hacer o aprender en un momento determinado, son útiles para seleccionar y establecer las secuencias de los contenidos que se pretenden enseñar" (Coll \& Martí, 1990, p. 359). Por tanto, las secuencias incluidas en una determinada etapa educativa deberían relacionarse con los conocimientos previos y las experiencias de los alumnos, para garantizar la progresión en los procesos de aprendizaje que van desarrollando.

A partir de los antecedentes expuestos, se puede inferir que una de las dificultades de los procesos de enseñanza y aprendizaje de las ciencias es que los profesores no están secuenciando los contenidos a partir de las ideas previas, intereses, motivaciones y necesidades que pudiese tener el estudiantado.

Estudios realizados por Yang et al. (2016) demostraron que, en comparación con el enfoque tradicional de la enseñanza centrada en el profesor, la secuenciación de los contenidos a partir de los intereses de los estudiantes podría mejorar la participación y el rendimiento de aprendizaje de ellos. Al incluir a los estudiantes en una secuenciación pensada desde y para ellos, y no en un orden dado por la 
construcción científica o lo estrictamente señalado por el currículo y el libro de texto, la investigación de Yang et al. (2016) reporta cuatro beneficios principales: a) mejora la eficiencia docente, b) promueve el aprendizaje activo, c) facilita la comprensión del contenido y, d) mejora la autoeficacia y actitudes del estudiantado.

Desde esta premisa, nace la propuesta de secuenciar las actividades de enseñanza a partir de los intereses de los estudiantes, lo cual, debería influir sobre los aprendizajes y sobre sus percepciones con respecto a las ciencias. Dado esto, en este estudio se busca responder a la siguiente pregunta de investigación: ¿Cómo influye la secuenciación de actividades a partir de los intereses de los estudiantes, en sus percepciones y aprendizajes sobre la enseñanza de las ciencias?

Para responder a este interrogante se han planteado dos supuestos: a) la secuenciación de actividades a partir de los intereses de los estudiantes influye en las percepciones que ellos tienen sobre la enseñanza de las ciencias porque les permite pasar de una mirada lineal y academicista de la ciencia a una postura que relaciona el contenido científico con problemáticas sociales y temas de actualidad y b) la secuenciación de actividades a partir de los intereses de los estudiantes influye en los aprendizajes que ellos adquieren sobre nociones científicas específicas.

Considerando los supuestos planteados anteriormente, es necesario establecer una postura sobre cuál es la finalidad de la secuenciación de contenidos de enseñanza y las consideraciones que favorecen los aprendizajes de los estudiantes.

\section{FINALIDAD Y CONSIDERACIONES PARA LA SECUENCIACIÓN DE CONTENIDOS DE ENSEÑANZA}

La finalidad de la secuenciación de los contenidos de enseñanza es lograr una relación lógica entre los objetivos educativos y las actividades de aprendizaje de los estudiantes, alcanzando condiciones mínimas para transformar los ambientes de aprendizajes de la sala de clase, la comunidad educativa y la institución. Se debe tener en cuenta que para la secuenciación de contenidos es conveniente disponer de orientaciones teóricas que ayuden a escoger y estructurar los contenidos según criterios didácticos (Izquierdo, 2005; Zapata, 2005).

El profesor debe adquirir conocimientos fundamentados sobre la enseñanza de las ciencias: saber detectar, analizar e interpretar los intereses de los estudiantes con respecto a diferentes nociones científicas, prestando especial atención al análisis de los posibles obstáculos que impiden la construcción de las ideas de la ciencia y al establecimiento de posibles itinerarios para superarlos (Jack \& Lin, 2014; Krapp \& Prenzel, 2011, Palmer, 2009). Para comprender y favorecer el aprendizaje del estudiantado, es necesario conocer sus procesos psicológicos y sociológicos, lo que se ve reflejado cuando se consideran los siguientes aspectos para la secuenciación de actividades: a) atención a las ideas previas, b) atención a los obstáculos que impiden 
la construcción del aprendizaje, c) abrir nuevas perspectivas e integrar nuevos conocimientos que sean significativos para el estudiante, d) voluntad de motivar a toda clase de estudiantes y, e) conocer el contexto del estudiante (Adúriz-Bravo, Izquierdo \& Estany, 2002).

Dado que la selección, organización y secuenciación de los contenidos, son un desafío profesional para el profesorado, es preciso plantear cuatro orientaciones básicas que permiten replantear la enseñanza de las ciencias.

Lo primero que hay que tener en cuenta son los objetivos generales establecidos en el programa formativo, los cuales deben incidir en la acción formativa a través de la relación entre los objetivos generales y los contenidos establecidos para cada nivel (Gunstone, 2012). Por ende, los objetivos generales tendrán que incidir en la acción educativa a través de distintas áreas del currículo y de los contenidos de enseñanza, ya sean conceptuales, procedimentales o actitudinales.

Esto conlleva al profesorado a diseñar el proceso de enseñanza de cada una de las unidades, áreas, módulos o niveles, incluyendo tanto los contenidos como los objetivos de aprendizaje, así como también, los contextos de sus estudiantes que facilitan u obstaculizan el logro de los objetivos que se pretenden alcanzar (Sanmartí, 2002).

La segunda consideración está referida al estudiante, que en un caso óptimo va a desarrollar capacidades y habilidades que están asociadas a los contenidos de aprendizaje establecidos para el nivel correspondiente (Ainley \& Ainley, 2011; Potvin \& Hasni, 2014; Vedder-Weiss \& Fortus, 2011). Por lo tanto, es importante procurar que los objetivos de aprendizaje estén orientados hacia la formación integral de todos los estudiantes.

En tercer lugar, el rol del profesor se convierte en un acto intencionado que incide de manera directa sobre los procesos de enseñanza y aprendizaje; lo cual se traduce en las decisiones que tome con respecto a los criterios, estrategias de aprendizaje y elección de materiales curriculares que conlleven a la construcción del conocimiento por parte del estudiantado (Renninger \& Hidi, 2011; Sanmartí, 2002; Schneider \& Plasman, 2011; Vanegas-Ortega, 2018; Wilson, 2013).

En cuarto lugar, el profesorado debe estructurar sus procesos de enseñanza, secuenciando el contenido y diseñando actividades que respondan a las características del estudiantado, debido a que es el único que puede llegar a saber sobre las capacidades e intereses de ellos (Dewitt et al., 2013; Vedder-Weiss \& Fortus, 2011; 2019). De esa manera, podrá lograr atender la diversidad, asegurando la apropiación de conceptos, procedimientos básicos y promoviendo el desarrollo de cada estudiante.

Si el profesorado comparte criterios similares a los cuatro mencionados anteriormente, se podría lograr un trabajo en equipo que acerque el conocimiento científico a los contextos socioculturales de los 
estudiantes. Sin embargo, esto exige repensar los roles de los profesores y de los estudiantes frente a la secuenciación de contenido.

Un desafío importante para los profesores es acercar a los estudiantes al conocimiento de las ciencias, que "es el conjunto de conocimientos a enseñar y de aprendizajes a construir a través de una educación científica formal, sistemática y organizada, en donde los estudiantes alcancen niveles deseables de alfabetización científica, lo cual se puede lograr a partir de las tres dimensiones de los contenidos: el teórico, el procesual y el actitudinal, que se estructura en el modelo actual de ciencias" (Liguori \& Noste, 2005, p. 5). Para que ese acercamiento se concrete, resulta importante que el contenido a enseñar y las actividades a diseñar, se secuencien de tal manera que sea asimilable para los estudiantes y que atienda a sus intereses (Krapp \& Prenzel, 2011).

El profesor debe desempeñar un nuevo rol y estar capacitado para desarrollar un currículo distinto (no lineal), esto implica diseñar unidades didácticas a través del trabajo cooperativo, considerar aspectos para estructurar una clase mediante criterios claros para la selección y secuenciación de contenidos, tener claridad de los objetivos de aprendizaje que se quieren lograr y qué modelos didácticos considerar para conocer las dificultades de los estudiantes en el proceso de aprendizaje (Mazo \& Bonilla, 2021; Daza Et Al., 2014).

Aunque el profesor tiene la responsabilidad profesional de secuenciar y seleccionar los contenidos de la disciplina de acuerdo con la lógica de la ciencia, la consideración del contexto de los estudiantes ofrece una perspectiva colaborativa y participativa en la que el estudiantado pueda manifestar sus intereses con respecto a temas científicos, y al mismo tiempo, le entregue lineamientos al profesor sobre formas de secuenciar los contenidos mediante actividades que respondan a sus expectativas y motivaciones (Ainley \& Ainley, 2011, Márquez, Roca \& Sanmartí, 2019; Vedder-Weiss \& Fortus, 2019).

\section{OBJETIVOS}

Los objetivos específicos de la investigación son:

- $\quad$ Identificar los intereses que tienen los estudiantes de enseñanza básica y media con respecto a un contenido específico correspondiente a cada nivel.

- Describir los resultados de aprendizaje de nociones científicas específicas que son enseñadas a partir de los intereses de los estudiantes de básica y media.

- Determinar cambios en las percepciones de los estudiantes sobre la enseñanza de las ciencias, cuando aprenden nociones científicas específicas secuenciadas a partir de sus intereses.

\section{POBLACIÓN Y MUESTRA}

Los sujetos fueron seleccionados mediante la técnica de muestreo intencionado (Ruiz, 2012), puesto que, en coherencia con los fundamentos de la investigación-acción (Sandín, 2003), se trabajó con el estudiantado de una de las investigadoras. La población corresponde a estudiantes de los cursos de $5^{\circ}$ básico 
(10-11 años), $7^{\circ}$ básico (12-13 años), y $1^{\circ}$ año medio (14-15 años) $)^{1}$, que en total son 166 estudiantes, pertenecientes a un colegio particular subvencionado ubicado en la comuna de Renca de la Región Metropolitana de Chile. Esta institución posee una matrícula y arancel gratuita, además de un ingreso mixto con relación a los estudiantes, que se podría dividir en aproximadamente $60 \%$ mujeres y $40 \%$ hombres. Además, es importante señalar que a partir de los protocolos éticos de la investigación se solicitó el consentimiento informado de los padres o apoderados y el asentimiento de los estudiantes.

\section{INSTRUMENTO}

Dado que la investigación cuenta con tres fases de aplicación, para la primera fase se utilizó una actividad de aula para detectar intereses de los estudiantes, el cual consiste en la presentación de imágenes (cada imagen representa un indicador de evaluación del currículo correspondiente a cada nivel, ejemplificando las nociones científicas especificas a tratar), tras la observación de las imágenes los estudiantes responden un cuestionario de 7 preguntas abiertas, en donde se logró identificar los intereses del estudiantado. Las unidades abordadas para cada nivel fueron: $5^{\circ}$ Básico “¿Cómo afecta la alimentación en la salud de las personas?"; $7^{\circ}$ Básico "Fuerzas y ciencias de la tierra" y; $1^{\circ}$ Medio "Organismos en ecosistemas”. Este instrumento permitió construir la secuenciación de las actividades de enseñanza según los intereses de los estudiantes.

Además, se aplicó un instrumento con 8 preguntas cerradas y 1 pregunta abierta, que buscó identificar las percepciones de los estudiantes sobre las ciencias y las clases de ciencias. Los instrumentos utilizados en la fase uno, fueron revisados y validados por investigadores en didácticas de las ciencias, estos juicios de expertos permitieron garantizar la validez y confiabilidad de la información recogida (Cabero Almenara \& Llorente Cejudo, 2013).

La segunda fase consistió en la aplicación de las secuencias construidas a partir de los intereses y percepciones de los estudiantes, relacionado con el objetivo de aprendizaje y los indicadores de evaluación asociados a cada imagen del instrumento aplicado en la primera fase. Para ello se diseñó una unidad didáctica correspondiente a cada nivel, las cuales constan de cinco sesiones. Para poder evaluar si la secuencia implementada favoreció los aprendizajes científicos específicos de cada nivel, se realizó una evaluación final basada en la síntesis o aplicación de los conocimientos correspondientes, estos procesos evaluativos consideraron diferentes estrategias: en $5^{\circ}$ básico una exposición oral, en $7^{\circ}$ básico el análisis de un video y, en $1^{\circ}$ medio el análisis de casos. Estas actividades finales evaluaron el aprendizaje alcanzado mediante rúbricas diseñadas por los investigadores.

\footnotetext{
${ }^{1}$ Niveles del Sistema escolar Chileno.
} 
La tercera fase, se consolida mediante el instrumento que identificó las percepciones de los estudiantes sobre las ciencias y las clases de ciencias, se utilizó en dos momentos, antes (fase 1) y después (fase 3) de la implementación de las secuencias de actividades.

\section{PROCEDIMIENTO DE RECOGIDA Y ANÁLISIS DE DATOS}

En esta investigación se utilizó una metodología mixta, debido a la integración descriptiva del paradigma cuantitativo y cualitativo en diversos aspectos metodológicos, lo cual contempla las ventajas de ambos enfoques (Hernández, Fernández \& Baptista, 2003), esto conlleva un gran nivel de complementación, ya que poder incorporar datos cuantitativos y aspectos cualitativos tales como narraciones, imágenes, verbalizaciones y percepciones de los actores.

La combinación de la cuantificación descriptiva y la cualificación interpretativa ofrece un mayor sentido a la investigación, en especial cuando los estudios son enfocados a ámbitos de la educación (Esterberg, 2002; Pereira Pérez, 2011). Este enfoque permitió, desde el punto de vista cualitativo, explicar la influencia de la secuenciación de actividades a partir de los intereses de los estudiantes, en sus percepciones sobre la enseñanza de las ciencias y en los aprendizajes de nociones científicas específicas, y desde la mirada cuantitativa, generar un alcance descriptivo para observar algunas regularidades en los resultados obtenidos (Hart, Smith, Swars \& Smith, 2009).

El diseño se ha estructurado siguiendo los lineamientos de la Investigación-Acción (Esterberg, 2002; Sandín, 2003 \& Stringer, 2007), puesto que se pretendió generar conocimiento mediante y para la práctica pedagógica del profesorado de ciencias. Esto significó la transformación y mejora de la realidad de las salas de clase de los estudiantes con los que se aplicaron las unidades didácticas, examinando constantemente sus intereses, percepciones y aprendizajes sobre las nociones científicas específicas de cada nivel.

Como se muestra en la figura 1, el diseño se estructuró coherentemente con los objetivos específicos de la investigación. La producción de datos inicia y finaliza con la aplicación del instrumento de percepciones, lo que permite dar cuenta del tercer objetivo de la investigación.

La primera fase de la investigación estuvo dedicada a identificar los intereses que tienen los estudiantes de enseñanza básica y media con respecto a nociones científicas específicas correspondiente a cada nivel, para ello se aplicó el instrumento de intereses el cual constaba de 7 preguntas abiertas, las que fueron agrupadas por los investigadores en: a) no responde la pregunta, b) nociones científicas relacionadas a la vida cotidiana y c) nociones científicas específicas. Esto orientó el enfoque de la secuenciación de actividades con base en los intereses de los estudiantes. 
Figura 1. Diseño de la investigación según los objetivos específicos

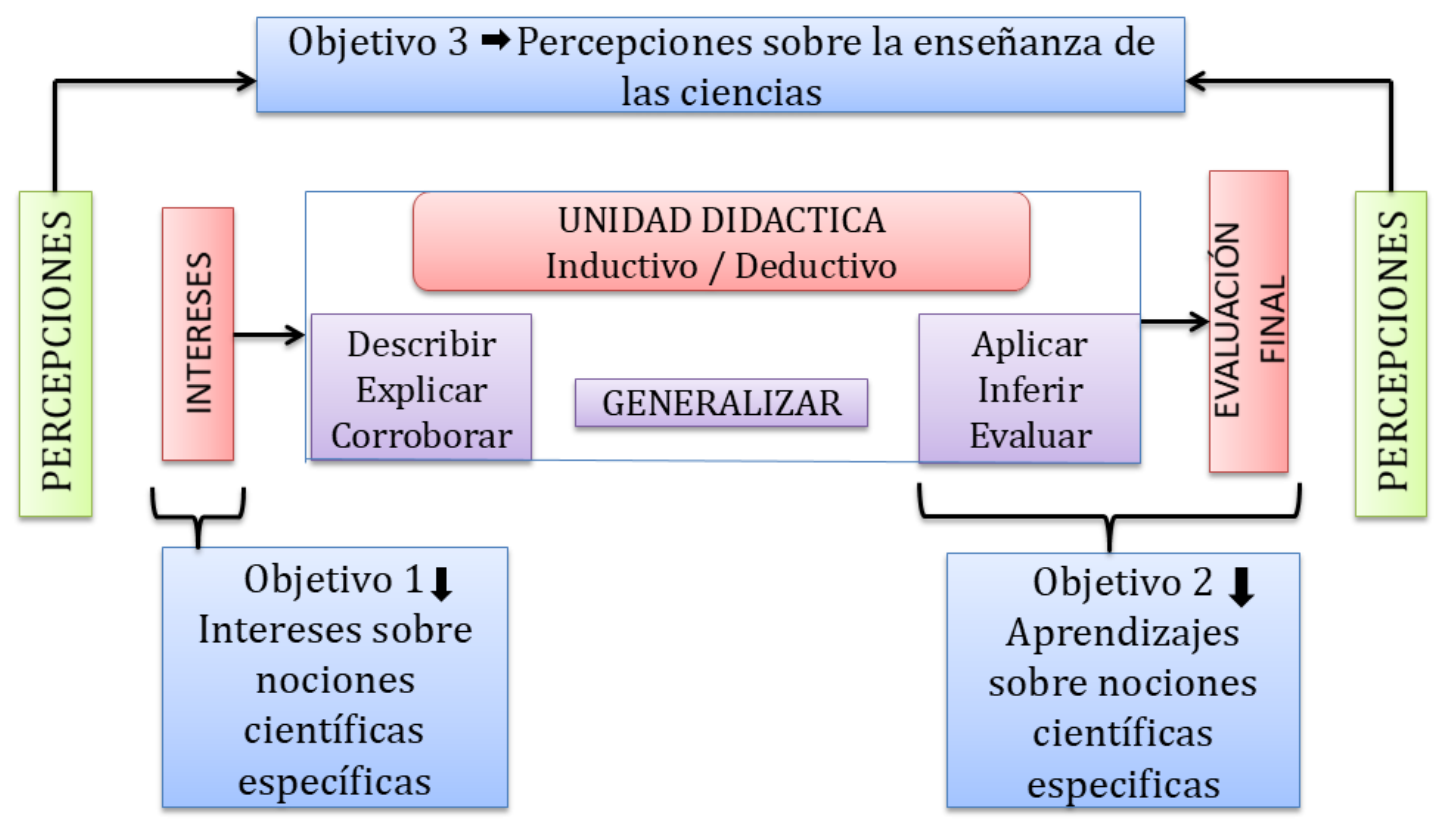

Fuente: Elaboración propia.

La segunda fase de la investigación buscó describir los resultados de aprendizaje de nociones científicas específicas que son enseñadas a partir de los intereses de los estudiantes. Esto comprende la aplicación de una unidad didáctica diseñada desde la propuesta teórica de Márquez y Roca (2009); se planificaron 5 clases por nivel, 4 clases de actividades sobre nociones científicas específicas y una clase de actividad de evaluación final con calificación.

En la tercera fase de la investigación, con base en los resultados del instrumento de percepciones, se determinaron cambios en las percepciones de los estudiantes sobre la enseñanza de las ciencias cuando aprenden nociones científicas específicas secuenciadas a partir de sus intereses. Los análisis se realizaron mediante la comparación de los resultados obtenidos en el inicio (fase 1) y al final de la secuenciación (fase $3)$.

Los datos cuantitativos, de naturaleza descriptiva, se agruparon en tablas y gráficos para determinar regularidades sobre las percepciones, intereses y aprendizajes de los estudiantes. Los datos cualitativos se documentan integrando los diferentes puntos de vista de los estudiantes y fueron reducidos mediante procesos de triangulación entre investigadores (Vallejo \& Finol De Franco, 2010).

\section{RESULTADOS}

\subsection{LA COTIDIANIDAD COMO INSUMO PARA LOS INTERESES DE LOS ESTUDIANTES}

De acuerdo con los resultados obtenidos de los instrumentos de intereses aplicado a los estudiantes, se puede apreciar las relaciones que se establecieron entre las nociones científicas específicas correspondientes a cada curso y las palabras más utilizadas por los mismos aprendices, con el fin de evaluar 
qué conceptos son los más pertinentes para abordar las unidades. En las figuras 2, 3 y 4 se sintetizan los principales hallazgos:

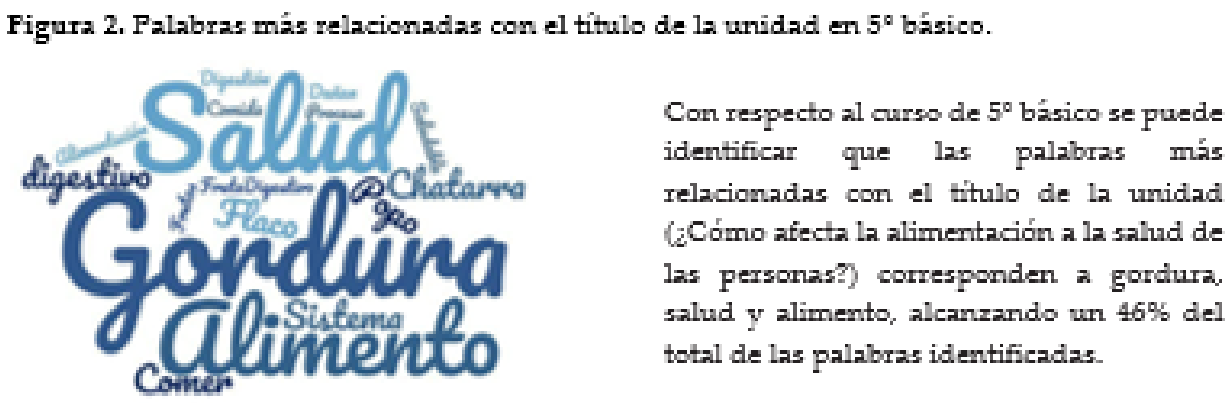

Fuente: Elaboración propia.

Figura 3. Palabras más relacionadas con el título de la unidad en $7^{\circ}$ básico

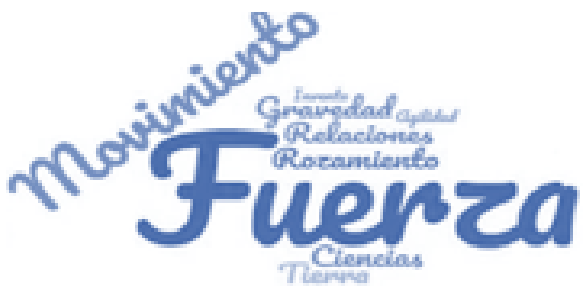

Con respecto al curso de $7^{\circ}$ básico se puede identificar que las palabras más relacionadas con el título de la unidad (Fuerza y ciencias de la tierra) fueron fuerza, movimiento $y$ ciencias, alcanzando un $77 \%$ del total de las palabras identificadas.

Fuente: Elaboración propia.

Figura 4. Palabras más relacionadas con el titulo de la unidad en $1^{\circ}$ medio.

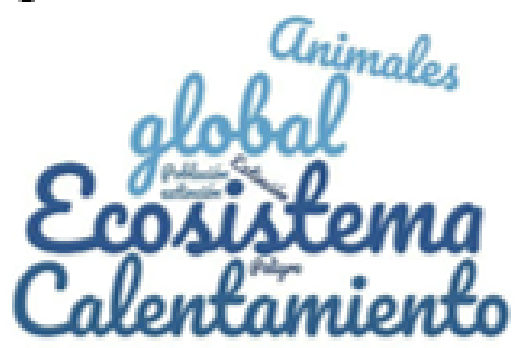

Con respecto al curso de $1^{\circ}$ medio se puede identificar que las palabras más relacionadas con el titulo de la unidad (Organismos en ecosistemas) son ecosistema, calentamiento y global, alcanzando un $69 \%$ del total de palabras identificadas.

Fuente: Elaboración propia.

Estos resultados revelan que los estudiantes de distintos niveles asocian el contenido disciplinar con sus experiencias cotidianas (Dewitt et al., 2013). Por ejemplo, en $5^{\circ}$ básico observar imágenes de alimentos saludables versus otros que no lo son, lo relacionan con salud; y en $7^{\circ}$ básico al observar una persona en patines inmediatamente surge la idea de movimiento como algo natural, sin analizar físicamente que es un cambio de la posición de un cuerpo a lo largo del tiempo respecto de un sistema de referencia. Según Sanmartí (2002), el profesorado debe considerar al momento de secuenciar, las características del estudiantado y las experiencias previas, para así promover el desarrollo de cada estudiante. Es por esto que 
los resultados permitieron considerar elementos de la cotidianidad de los estudiantes al momento de secuenciar y crear las actividades de cada unidad didáctica

\subsection{USO DE IMÁGENES PARA PERFILAR LOS INTERESES HACIA NOCIONES CIENTÍFICAS ESPECÍFICAS}

Tras la aplicación del instrumento de intereses antes de la secuenciación de actividades de aprendizaje, los estudiantes declararon qué imagen podrían explicar con mayor facilidad y cuál no.

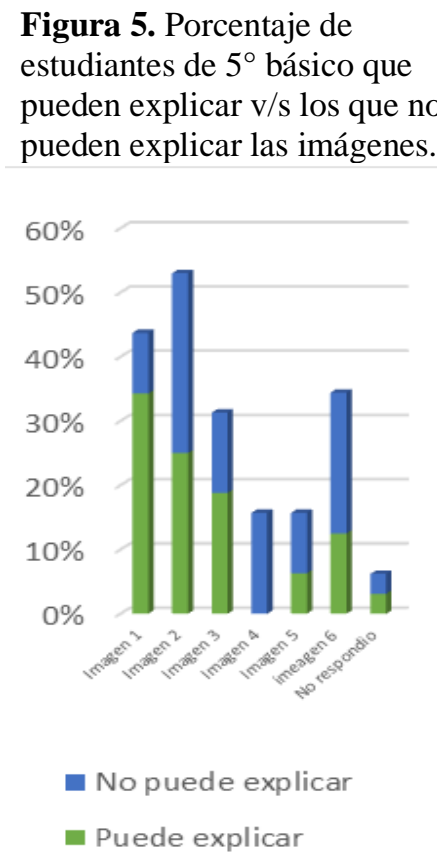

Fuente: Elaboración propia.
Figura 6. Porcentaje de estudiantes de $7^{\circ}$ básico que pueden explicar v/s los que no pueden explicar las imágenes.
Figura 7. Porcentaje de estudiantes de $1^{\circ}$ medio que pueden explicar v/s los que no pueden explicar las imágenes.

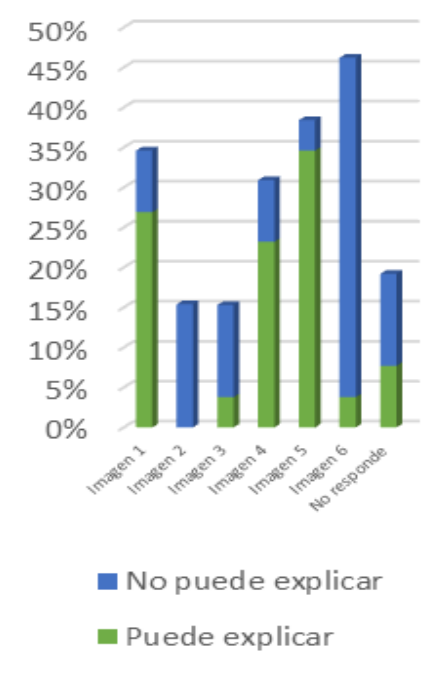

Fuente: Elaboración propia.

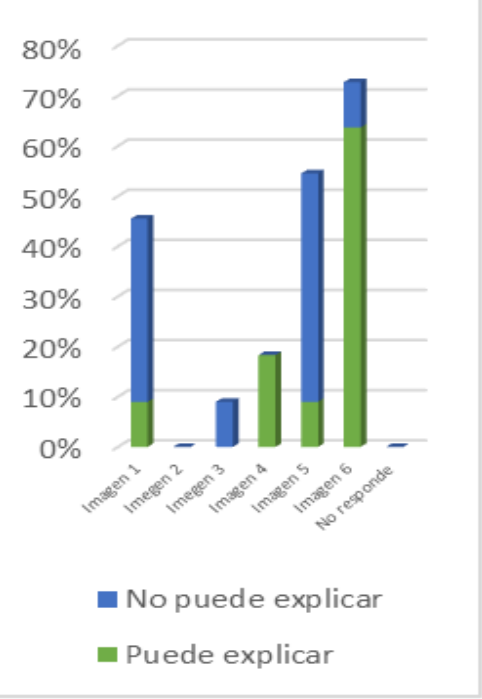

Fuente: Elaboración propia.

Según la figura 5 , en $5^{\circ}$ básico los estudiantes declaran poder explicar con mayor facilidad a un compañero la imagen número 1, que corresponde al indicador de evaluación: "Relacionan los distintos grupos de alimentos con el aporte especifico que realizan al cuerpo (IE 6)". Mientras que la imagen que no pueden explicar a un compañero es la número 2, que corresponde al indicador de evaluación: "Predicen posibles efectos de una baja ingesta de alimentos en el funcionamiento corporal (IE 4)".

Según la figura 6 , en $7^{\circ}$ básico los estudiantes declaran poder explicar con mayor facilidad a un compañero la imagen número 5, que corresponde al indicador de evaluación: "Describen el efecto de la presión entre sólidos, como ocurre en herramientas de uso cotidiano (IE 2)". Mientras que la imagen que no pueden explicar a un compañero es la número 6, que corresponde al indicador de evaluación "Explican, cualitativamente, que la presión hidrostática en un fluido depende de la profundidad. (IE 3)”.

Según la figura 7 , en $1^{\circ}$ medio los estudiantes declaran poder explicar con mayor facilidad a un compañero la imagen número 6, que corresponde al indicador de evaluación: "Discuten posibles 
consecuencias de la extinción de especies o poblaciones sobre las funciones ecosistémicas considerando los seres humanos (IE 5)". Mientras que la imagen que no pueden explicar a un compañero es la número 5, que corresponde al indicador de evaluación "Analizan factores antrópicos y naturales que pueden afectar el tamaño de las poblaciones en situaciones de fenómenos del cambio climático, epidemias y pandemias, disponibilidad de recursos energéticos o alimentarios y cambio del uso del suelo, entre otras. (IE 3)".

\subsection{SECUENCIACIÓN SEGÚN LOS INTERESES DE LOS ESTUDIANTES}

Para determinar la secuenciación de actividades didácticas, primero fue necesario ordenar el contenido de acuerdo a los intereses de los estudiantes, el cual se determinó mediante la cantidad de preguntas que se plantearon al observar las imágenes. Además, en grupos de trabajo ordenaron de mayor a menor interés las preguntas que les llamó más la atención y les gustaría responder en la clase de ciencias.

La tabla 1 muestra el orden de las imágenes según los intereses de los estudiantes (de mayor a menor interés). Considerando que cada imagen representa un indicador de evaluación (IE) y un objetivo de aprendizaje (OA) de las nociones científicas específicas correspondiente a cada nivel.

Tabla 1. Orden de las imágenes según el interés de los estudiantes (de mayor a menor interés).

\begin{tabular}{|l|l|l|}
\hline $\mathbf{5}^{\circ}$ Básico & $\mathbf{7}^{\circ}$ Básico & $\mathbf{1}^{\circ}$ Medio \\
\hline Alimentación & Gravedad & Niveles tróficos \\
\hline Extremos alimenticios & Elasticidad & Interacciones \\
\hline Variedad & Ley Hooke & Población \\
\hline Digestión & Movimiento & Extinción \\
\hline Sistema digestivo & Presión & Hábitat \\
\hline Defecación & Hidrostática & Calentamiento \\
\hline
\end{tabular}

\subsection{NIVEL DE APRENDIZAJES ALCANZADOS EN LA EVALUACIÓN FINAL}

A continuación se presentan los resultados obtenidos luego de la aplicación de la secuenciación de actividades didácticas. En la figura 8 se observa el nivel de aprendizaje alcanzado por los diferentes cursos durante la evaluación final, es decir, al término de la aplicación de la secuenciación de actividades según los intereses de los estudiantes.

Los resultados obtenidos indican que la mayoría de los estudiantes de los tres niveles, lograron los aprendizajes sobre las nociones científicas específicas. En menor proporción, algunos tuvieron aprendizajes medianamente logrados (notas de 4,0 a 5,4). Se destaca el trabajo realizado en $1^{\circ}$ medio puesto que ninguno de los estudiantes obtuvo calificaciones por debajo de la valoración de aprobación $(4,0)$. En los cursos de educación básica, aunque hay algunos estudiantes con valoración de aprendizajes no logrados, se trata de un porcentaje mínimo en comparación con los resultados generales. 
Figura 8. Logro alcanzado por estudiantes en evaluación final. (No logrado: nota 2,0 a 3,9. Medianamente logrado: nota 4,0 a 5,4. Logrado: nota 5,5 a 7,0.)

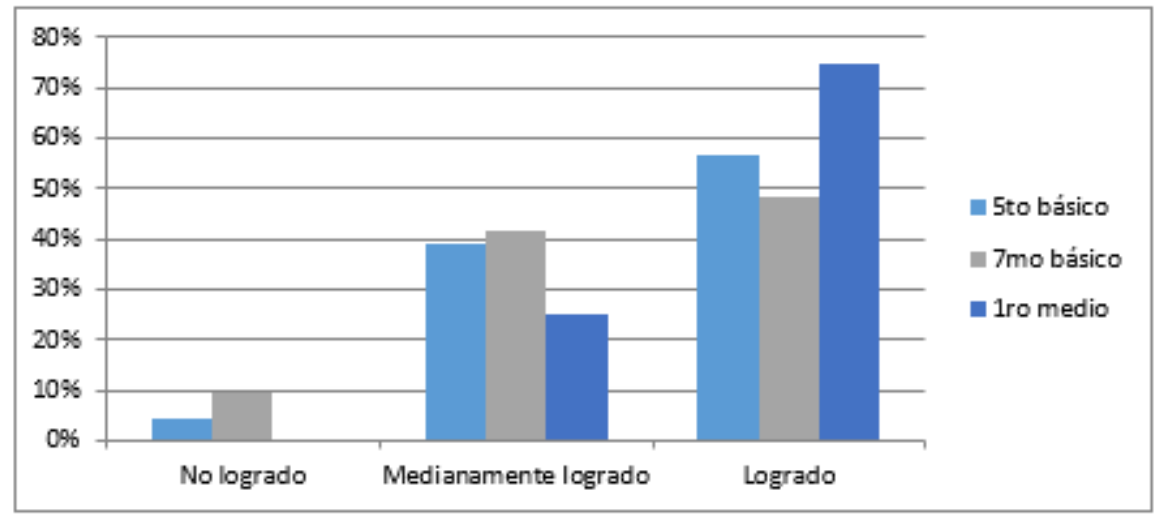

\subsection{DECLARACIÓN DE LOS ESTUDIANTES DE SUS APRENDIZAJES EN LA ACTIVIDAD} FINAL

Los estudiantes declararon sus aprendizajes sobre nociones científicas específicas de cada nivel, obteniendo los resultados sintetizados en la tabla 2.

Tabla 2. Declaración de los estudiantes sobre sus aprendizajes.

\begin{tabular}{|l|l|l|l|l|l|}
\hline & $\begin{array}{l}\text { Lo entiendo y } \\
\text { puedo } \\
\text { explicar a otra } \\
\text { persona }\end{array}$ & $\begin{array}{l}\text { Lo entiendo, } \\
\text { pero no podría } \\
\text { explicar a otra } \\
\text { persona }\end{array}$ & $\begin{array}{l}\text { Sé de qué se } \\
\text { trata, pero no } \\
\text { lo entiendo }\end{array}$ & No la conozco & Otra Opción \\
\hline $5^{\circ}$ Básico & $35.94 \%$ & $31.77 \%$ & $21.88 \%$ & $6.77 \%$ & $3.65 \%$ \\
\hline $7^{\circ}$ Básico & $31.57 \%$ & $47.30 \%$ & $11.80 \%$ & $9.10 \%$ & $0.00 \%$ \\
\hline $1^{\circ}$ Medio & $36.67 \%$ & $35.50 \%$ & $27.50 \%$ & $2.50 \%$ & $0.83 \%$ \\
\hline
\end{tabular}

Fuente: Elaboración propia

Estos resultados permiten destacar que en $5^{\circ}$ básico y $1^{\circ}$ medio la mayoría de los estudiantes declaran el nivel más alto de aprendizaje de la escala (lo entiende y lo puede explicar a otra persona). Sin embargo, en $7^{\circ}$ básico, el más alto porcentaje del estudiantado declara que si bien entiende las situaciones presentadas, no logran explicarlo a otra persona.

Con el fin de establecer relaciones entre los aprendizajes reales alcanzados por los estudiantes en la evaluación final (valoraciones iguales o superiores a 4.0) y los aprendizajes que ellos declaran haber obtenido, los datos de la figura 10 se pueden agrupar en dos grandes criterios: a) Declaración de haber aprendido sobre la noción científica específica, donde se incluye "La entiendo y puedo explicar a otra persona" y "La entiendo pero no podría explicar a otra persona"; b) Declaración de no haber aprendido sobre la noción científica específica, donde se incluye "Sé de qué se trata pero no la entiendo" y "No la conozco".

A partir de lo anterior, y relacionando la información de las figuras 8 y 9, se puede establecer que:

- $\quad$ En $5^{\circ}$ básico: el 95,70\% de los estudiantes lograron los aprendizajes esperados, mientras que la percepción de ellos es que el 67,71\% lograron los aprendizajes abordados en la unidad didáctica. 
- $\quad$ En $7^{\circ}$ básico: el 90,40\% de los estudiantes lograron los aprendizajes esperados, mientras que la percepción de ellos es que el 79,00\% lograron los aprendizajes abordados en la unidad didáctica.

- $\quad$ En $1^{\circ}$ medio: el $100 \%$ de los estudiantes lograron los aprendizajes esperados, mientras que la percepción de ellos es que el 72,17\% lograron los aprendizajes abordados en la unidad didáctica.

Figura 9. Declaración de los estudiantes sobre sus aprendizajes.

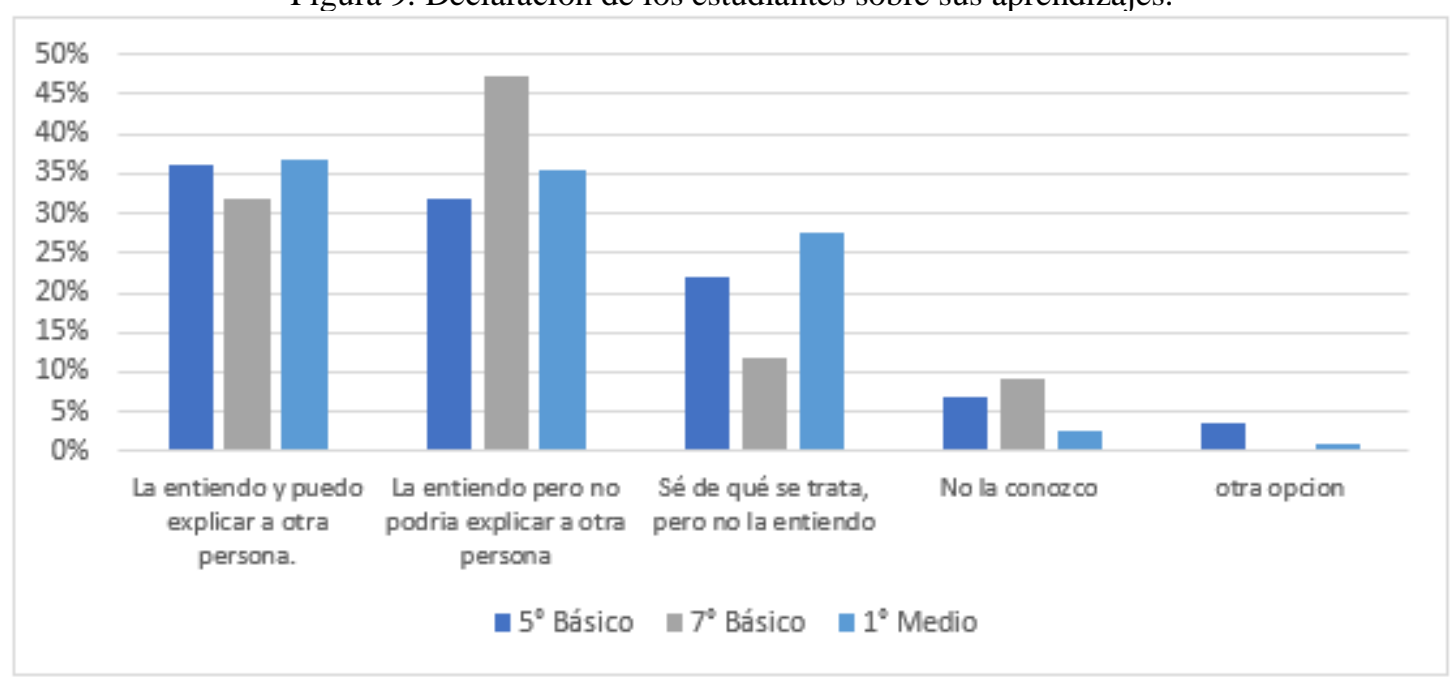

Fuente: Elaboración propia

Esta comparación indica que, aunque la mayoría de los estudiantes perciben entender las nociones científicas específicas, las percepciones sobre sus aprendizajes son inferiores a los resultados de aprendizajes obtenidos en la evaluación final. Por tanto, es necesario dar continuidad a la implementación de actividades metacognitivas que permitan estrechar las diferencias entre este tipo de resultados.

Por otro lado, cuando a los estudiantes se les preguntó sobre las habilidades asociadas a la noción científica específica, se encontró que son capaces de desarrollar diferentes habilidades científicas dependiendo de la imagen que se presenta. Por ejemplo, como muestra la figura 10 , en $1^{\circ}$ medio el $39,17 \%$ puede analizar alguna situación con respecto al contenido, siendo una de las habilidades alcanzadas por mayor cantidad de estudiantes; esto concuerda con las habilidades de orden superior que se esperan que los estudiantes alcancen en el nivel de $1^{\circ}$ medio según los planes y programas vigentes en el módulo de biología. 
Figura 10. Habilidades que los estudiantes podrían aplicar mejor sobre el contenido.

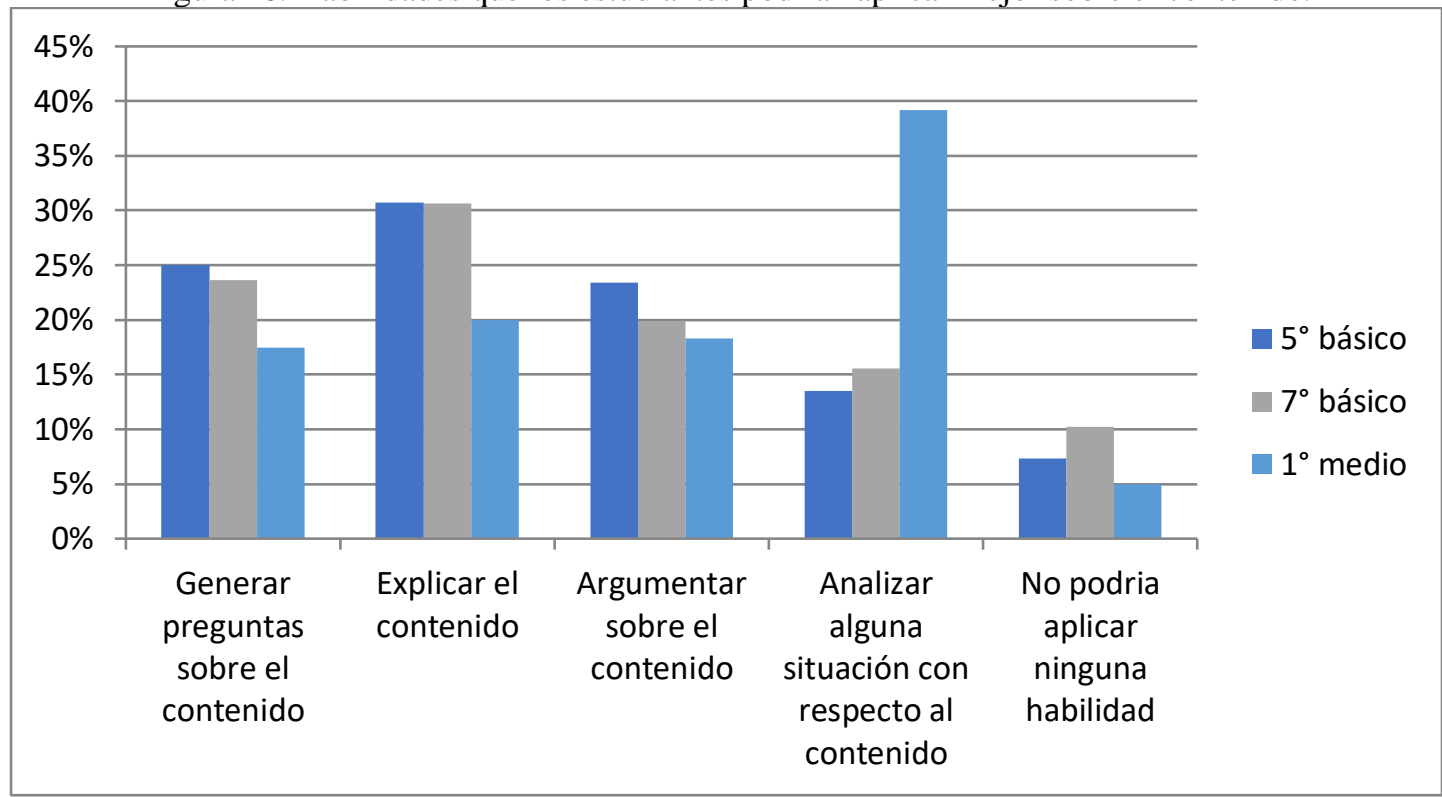

Fuente: Elaboración propia

Asimismo, cuando se les solicitó relacionar las imágenes de cada indicador de evaluación con la pregunta científica correspondiente, se encontró que gran parte de los estudiantes lograron relacionar el indicador de evaluación (imagen) con la pregunta correspondiente. Como se indica en la figura 11, el curso que en mayor porcentaje logró relaciones correctas fue $1^{\circ}$ medio $(65 \%)$, mientras los cursos de $5^{\circ}$ básico $(57,2 \%)$ y $7^{\circ}$ básico $(49,4 \%)$ tuvieron logros menores.

Figura 11. Relación entre indicador de evaluación (imagen) y pregunta correcta.

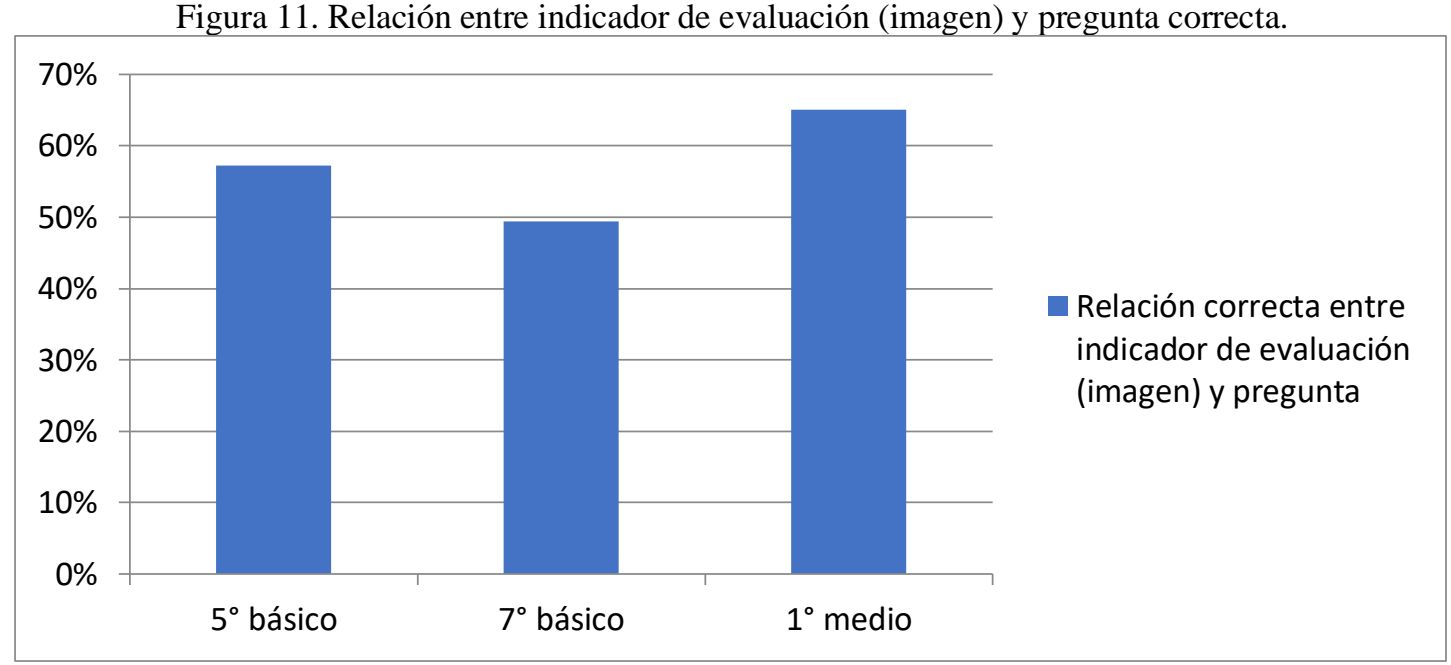

Fuente: Elaboración propia

\subsection{CAMBIOS EN LAS PERCEPCIONES SOBRE LA CIENCIA Y LAS CLASES DE CIENCIAS}

Los estudiantes declararon antes de la intervención que, en un 50,0\% les interesa mucho aprender sobre los hechos naturales y las ciencias en la sala de clases; un 55,3\% piensa que las ciencias les ayudan y les beneficia para su vida diaria; y a un 58,6\% le gusta la clase de ciencia. 
En comparación (figura 12), al finalizar la secuenciación, se aplicó el mismo instrumento el cual permitió recoger la siguiente información: En un 72,0\% les interesa mucho aprender sobre los hechos naturales y las ciencias en la sala de clase; un 69,0\% piensa que las ciencias les ayuda y les beneficia para su vida diaria; y un 79,3\% declara que les gusta la clase de ciencia. Estos resultados coinciden con el estudio realizado por Yang et al. (2016), ratificando que al incluir a los estudiantes en una secuenciación pensada desde y para ellos, mejora la participación y actitudes de los estudiantes por la ciencia y la clase de ciencias.

Figura 12. Percepciones de los estudiantes con respecto a la ciencia y las clases de ciencia al inicio y al final de la intervención

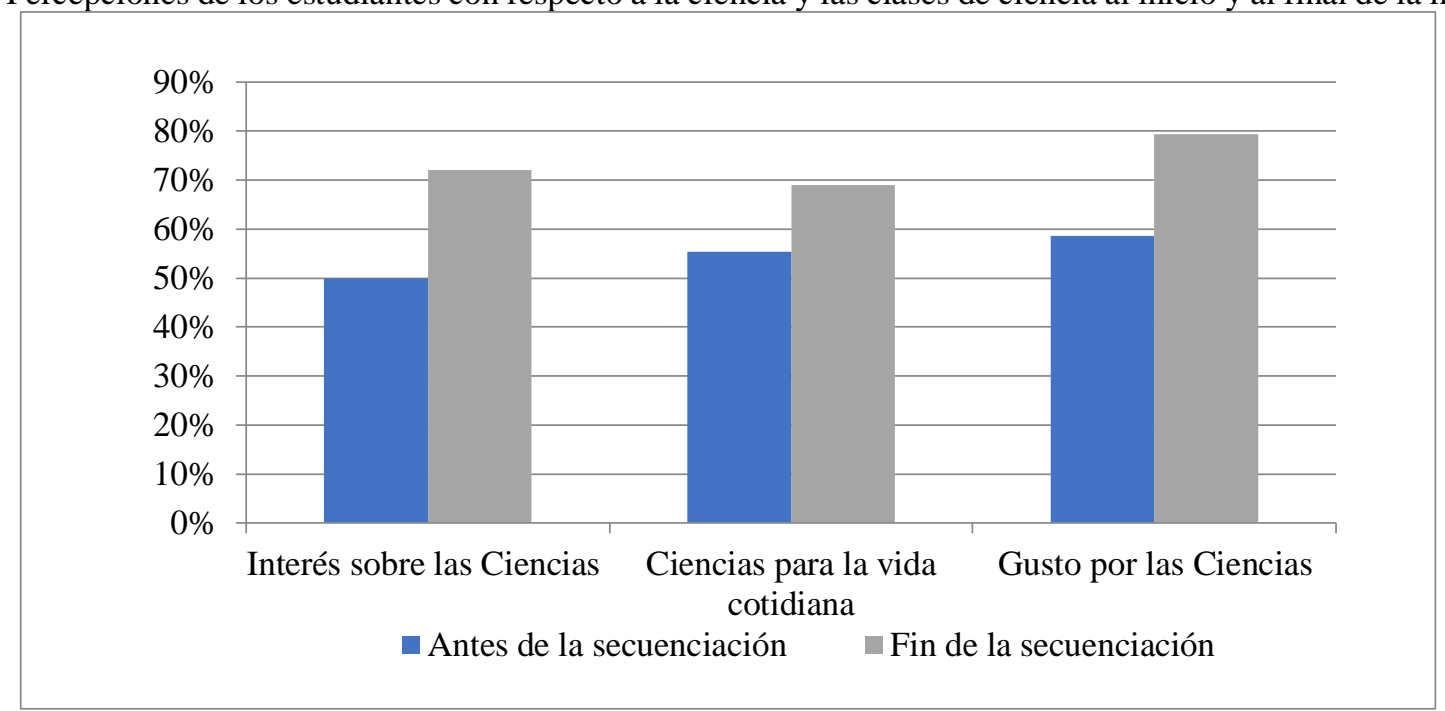

Fuente: Elaboración propia

El instrumento también permitió identificar las recomendaciones de los estudiantes para mejorar las clases de ciencias, las cuales se agruparon en las siguientes tres categorías:

a) Aspectos generales: los estudiantes recomiendan clases experimentales y más entretenidas.

b) Aspectos disposicionales: los estudiantes declaran que ellos deben tener un mejor comportamiento en la sala de clases y que es necesario la profesora a cargo mejore la forma de interrelacionarse con ellos.

c) Acciones concretas: los estudiantes declaran que les interesa hacer afiches, guías y exposiciones.

En los resultados de la aplicación final del instrumento, se resalta la autocrítica que demuestran los estudiantes en cuanto a la categoría de aspectos disposicionales, ya que declaran que ellos deben mejorar su comportamiento, y ya no se refieren a los factores asociados a la relación con la profesora. 


\section{DISCUSIÓN Y CONCLUSIONES}

Al final de la unidad didáctica, la evaluación final permitió concluir que hay un alto porcentaje de estudiantes que lograron los aprendizajes propuestos para cada una de las nociones científicas específicas, lo cual fue posible gracias la secuenciación de actividades a partir de los intereses de los estudiantes, sus ideas previas y contexto (Gunstone, 2012), además del conocimiento didáctico de contenido que se requirió para la construcción de las unidades didácticas (Abell \& Lederman, 2007; Cofré et al., 2010; Vanegas-Ortega, 2018).

Los estudiantes relacionan el contenido con las analogías que les son más cercanas, esto se demostró en el instrumento de intereses al vincular las imágenes con el título de la unidad. Siguiendo esta misma línea, al preguntarles a los estudiantes qué imagen podrían explicar con mayor facilidad a un compañero, ellos apuntan a aquella imagen que les es más sencillo describir, que es la primera fase para llegar a relacionar los componentes que forman un fenómeno y así llegar a una explicación científica (Márquez \& Roca, 2009; Mazo \& Bonilla, 2021).

La importancia del uso de imágenes para que los estudiantes manifiesten sus intereses radicó en que estas son un sistema externo de representación, que les permitió representarse al mundo, realizando una tarea cognitiva que les permitía con mayor facilidad inferir y establecer un significado (Lombardi, Caballero \& Moreira, 2009), por tanto, en esta investigación fue fundamental el uso de imágenes para conocer los intereses de los estudiantes, puesto que al asociarlas con su cotidianidad, pudieron generar preguntas y priorizarlas de acuerdo a sus intereses.

Como plantean Pérez-Echeverría, Martí y Pozo (2010), estas imágenes formaron un instrumento cognitivo, que constituye un conjunto de signos, reglas y código de composición, realizando una mediación entre las nociones científicas y el fenómeno del mundo real, generando posibilidades para interpretar, conocer, reinterpretar, redescribir y transformar el mundo. El uso de imágenes dirigidas, así como la elaboración de preguntas y su jerarquización, permitieron determinar la secuenciación de los contenidos según los intereses del estudiantado, y posteriormente, la construcción de secuencias de actividades que favorecieran los aprendizajes de los estudiantes.

Considerando que los intereses de los estudiantes fueron fundamentales para poder generar la secuenciación de actividades de aprendizaje, estos no son suficientes para diseñarla, puesto que es imprescindible contar con conocimientos didácticos del contenido (CDC), ya que como muestran otras investigaciones (Abell \& Lederman, 2007, Vanegas-Ortega, 2018), este tipo de formación del profesorado proporciona mayores niveles de aprendizajes en los estudiantes.

El CDC es un conocimiento específico, uno que va más allá del conocimiento de la disciplina (Shulman, 1986; Vergara \& Cofré, 2014; Vanegas-Ortega, 2018), el cual debe permitirle al profesorado 
facilitar las comprensiones de los estudiantes sobre conceptos y procedimientos específico mediante el diseño de estrategias y actividades que respondan al contexto, los aprendices y a la lógica de construcción de la disciplina (Jack \& Lin, 2014). Para lograr esto, es fundamental detectar los intereses de los estudiantes y articularlos con los contenidos establecidos en el currículo. El CDC permite que el profesorado tome decisiones conscientes y pertinentes para identificar los intereses de los estudiantes y secuenciar las actividades, logrando diseñar unidades didácticas vinculadas al contenido establecido por el currículo.

Después de aplicar la secuenciación de actividades de aprendizaje, se observó que los estudiantes lograron un alto nivel de aprendizaje y un autoreconocimiento del mismo. Esto demuestra que, en coherencia con Yang et al. (2016), la enseñanza desde los intereses de los estudiantes mejora la autoeficacia y las actitudes de los estudiantes hacia el aprendizaje de las ciencias naturales.

En cuanto a las percepciones de los estudiantes con respecto a las ciencias y las clases de ciencias, ellos demostraron al inicio de la investigación estar interesados en ellas, sin embargo, al final de la intervención sus percepciones mejoraron. Aunque los resultados de la investigación son favorables, se encontró que las percepciones de los estudiantes sobre sus aprendizajes aún no logran estar en correspondencia con lo que se deduce de las evaluaciones finales: los resultados de aprendizajes son mejores que las propias percepciones de logro. En ese sentido, no es suficiente la aplicación de una unidad didáctica para que los estudiantes aprendan a autorregular sus aprendizajes, sino que es necesario dar continuidad a una enseñanza de las ciencias basada en un proceso de co-creación entre el profesor y los estudiantes (Adúriz-Bravo, Izquierdo \& Estany, 2002; Izquierdo, 2005; Zapata, 2005).

Al comparar las recomendaciones realizadas por los estudiantes para mejorar la clase de ciencias, al inicio y al final de la implementación de la secuenciación de actividades de aprendizaje, estas no variaron en términos cuantitativos, sin embargo, desde el punto de vista cualitativo, ellos plantean al inicio, cambiar aspectos disposicionales de la profesora y del estudiantado, pero en sus percepciones finales declaran sólo mejoras en el comportamiento de ellos. Además de la autorregulación de los estudiantes (Gunstone, 2012; Izquierdo, 2005), esto da cuenta de cambios sucesivos que se fueron efectuados por la profesora que implementó las unidades didácticas, que combinado con los procesos de producción de datos y diseño didáctico, permiten inferir que la investigación también tuvo efectos sobre el desarrollo profesional de la docente (Wilson, 2013).

En síntesis, ¿cómo influye la secuenciación de actividades a partir de los intereses de los estudiantes, en sus percepciones y aprendizajes sobre la enseñanza de las ciencias? Mejoran las percepciones de los estudiantes, en cuanto al interés y gusto por las ciencias y las clases de ciencias, promoviendo la importancia de las ciencias para la vida cotidiana, además de facilitar el aprendizaje de 
las nociones científicas específicas. También, mejora la autoeficacia y actitudes del estudiantado hacia la clase de ciencias, enriqueciendo y proponiendo alternativas para mejorar las prácticas docentes. 


\section{REFERENCIAS}

ABELL, S; LEDERMAN, N. Handbook of research on science education. Mahwah, NJ: Lawrence Erlbaum Associates, 2007.

ADÚRIZ-BRAVO, A; IZQUIERDO M; ESTANY, A. Una propuesta para estructurar la enseñanza de la Filosofía de la Ciencia para el profesorado de Ciencias en formación. Enseñanza de las ciencias: Revista de investigación y experiencias didácticas. v. 20, n. 3, p. 465-476, 2002.

AINLEY, M., \& AINLEY, J. A cultural perspective on the structure of student interest in science. International Journal of Science Education, v.33, n. 1, p. 51-71, 2011.

CABERO ALMENARA, J; LLORENTE CEJUDO, M. La aplicación del juicio de experto como técnica de evaluación de las tecnologías de la información y comunicación (TIC). Revista de Tecnología de Información y Comunicación en Educación, v. 7, n. 2, p. 11-22, 2013.

COFRÉ, H; CAMACHO, J; GALAZ, A; JIMÉNEZ, J; SANTIBÁÑEZ, D; VERGARA, C. La educación científica en Chile: debilidades de la enseñanza y futuros desafíos de la educación de profesores de ciencia. Estudios pedagógicos (Valdivia), v. 36, n. 2, p. 279-293, 2010.

COLL, C; MARTÍ, E. Aprendizaje y desarrollo: la concepción genético-cognitiva del aprendizaje. En Coll, C; Palacios, J.; Marchesi, A. Desarrollo psicológico y educación II. Psicología de la educación. (pp. 121-139). Madrid, España: Alianza editorial, 1990.

DAZA. S; ARRIETA, J; RIOS, O. La selección, secuenciación y organización de los contenidos de biología desde el conocimiento didáctico del contenido (CDC) en los profesores de secundaria en Barrancabermeja. Ponencia presentada en la Tercera Conferencia Latinoamericana del International, History and Philosophy Of Science Teaching Group, Santiago, Chile, 2014.

DAZA. S., ARRIETA, J., \& RIOS, O. La selección, secuenciación y organización de los contenidos de biología desde el conocimiento didáctico del contenido (CDC) en los profesores de secundaria en Barrancabermeja. Ponencia presentada en la Tercera Conferencia Latinoamericana del International, History and Philosophy Of Science Teaching Group, Santiago, Chile, 2014

DEWITT, J; OSBORNE, J; ARCHER, L; DILLON, J; WILLIS, B; WONG, B. Young children's aspirations in science: The unequivocal, the uncertain and the unthinkable. International Journal of Science Education, v. 35, n. 6, p. 1037-1063, 2013.

DÍAZ-JATUF, J. Criterios de selección y organización de contenidos educativos para cursos y talleres en educación superior sobre Bibliotecología Social: una visión argentina. VI Jornadas de Bibliotecología “Usuarios del Siglo XXI: ¿cómo satisfacer sus demandas?” San Salvador de Jujuy, Argentina, 2019. ESTERBERG, K. Qualitative methods in social research. Massachusetts, Estado Unidos: McGraw Hill, 2002.

GUNSTONE, R. F. The importance of specific science content in the enhancement of metacognition. The content of science, v. 34, n. 2, p. 131-146, 2012.

HART, L; SMITH, S; SWARS, S.; SMITH, M. An examination of research methods in mathematics education (1995-2005). Journal of Mixed Methods Research, v. 3, n. 1, p. 26-41, 2009. 
HERNÁNDEZ, R; FERNÁNDEZ, C.; BAPTISTA, P. Metodología de la investigación (3a ed.). México: Mc Graw-Hill, 2003.

IZQUIERDO, M. Hacia una teoría de los contenidos escolares. Enseñanza de las ciencias: revista de investigación y experiencias didácticas, v. 23, n. 1, p. 111-122, 2005.

JACK, B; LIN, H. Igniting and sustaining interest among students who have grown cold toward science. Science Education, v. 98, n. 5, p. 792-814, 2014.

KRAPP, A., \& PRENZEL, M. Research on interest in science: Theories, methods, and findings. International journal of science education, v. 33, n. 1, p. 27-50, 2011.

LIGUORI, L., \& NOSTE, M. I. Didáctica de las ciencias naturales. Argentina, Rosario: Homo Sapiens, 2005

LOMBARDI, G; CABALLERO, C. Y MOREIRA, M. El concepto de representación externa como base teórica para generar estrategias que promuevan la lectura significativa del lenguaje científico. Revista de Investigación, v. 33, n. 66, p. 147-186, 2009.

MÁRQUEZ, C; ROCA, M; SANMARTÍ, N. Investigar en el campo de la Didáctica de las Ciencias: ¿Para qué? ¿Cómo? Revista Investigación en la Escuela, v. 69, p. 31-43, 2019.

MÁRQUEZ, C; ROCA, M. Plantear preguntas: un punto de partida para aprender ciencias. Revista Educación y pedagogía, v. 18, n. 45, 61-71, 2009.

MAZO, J., \& BONILLA, G. El uso de representaciones múltiples en clases de Ciencias Naturales para fortalecer la competencia argumentativa: The use of multiple representations in Natural Science classes to strengthen argumentative competence. South Florida Journal of Development, v. 2, n. 3, p. 46434651, 2021.

PALMER, D. Student interest generated during an inquiry skills lesson. Journal of Research in science Teaching, v. 46, n. 2, p. 147-165, 2009.

PENUEL, W; GALLAGHER, L; MOORTHY, S. Preparing teachers to design sequences of instruction in earth systems science: A comparison of three professional development programs. American Educational Research Journal, v. 48, n. 4, p. 996-1025, 2011.

PEREIRA PÉREZ, Z. Los diseños de método mixto en la investigación en educación: Una experiencia concreta. Revista Electrónica Educare, v. 15, n. 1, p. 15-29, 2011.

PÉREZ-ECHEVERRÍA, M, MARTÍ, E.; Pozo, J. Los sistemas externos de representación como herramientas de la mente. Cultura y Educación, v. 22, n. 2, p. 133-147, 2010.

POTVIN, P., \& HASNI, A. Interest, motivation and attitude towards science and technology at K-12 levels: a systematic review of 12 years of educational research. Studies in Science education, v. 50, n. 1, p. $85-129,2014$.

RENNINGER, K., \& HIDI, S. Revisiting the conceptualization, measurement, and generation of interest. Educational Psychologist, v. 46, n. 3, p. 168-184, 2011. 
RUIZ, J. Metodología de la investigación cualitativa. (5a ed.). Bilbao, España: [s.n], 2012.

SÁNCHEZ BLANCO G; VALCÁRCEL PÉREZ M. ¿Qué tienen en cuenta los profesores cuando seleccionan el contenido de enseñanza? Cambios y dificultades tras un programa de formación. Enseñanza de las ciencias: Revista de investigación y experiencias didácticas, v. 18, n. 3, p. 423-437, 2000.

SANDÍN, M. Investigación cualitativa en educación. Fundamentos y tradiciones. Madrid, España: McGraw-Hill, 2003.

SANMARTÍ, N. Didáctica de las ciencias en la educación secundaria obligatoria. Madrid, España: Sintesis, S.A, 2002.

SCHNEIDER, R., \& PLASMAN, K. Science teacher learning progressions: A review of science teachers' pedagogical content knowledge development. Review of Educational Research, v. 81, n. 4, p. 530-565, 2011.

SHULMAN, L. Those who understand: Knowledge Growth in Teaching. Educational Researcher, v. 15, n. 2, p. 4-14, 1986.

STRINGER, E. Action Research (3a Ed.). San Francisco, CA: Sage, 2007.

VALLEJO, R; FINOL DE FRANCO, M. La triangulación como procedimiento de análisis para investigaciones educativas. Revista electrónica de humanidades, educación y comunicación social, v. 7, n. 4, p. 117-133, 2010.

VANEGAS-ORTEGA, C. Visiones epistemológicas de ciencia y acción didáctica: correspondencias analógicas durante las prácticas pedagógicas de formación inicial de profesores. Revista de Innovación en Enseñanza de las Ciencias, v. 2, n. 2, p. 72-90, 2018.

VEDDER-WEISS, D., \& FORTUS, D. Adolescents' declining motivation to learn science: Inevitable or not? Journal of Research in Science Teaching, v. 48, n. 2, p. 199-216, 2011.

VEDDER-WEISS, D., \& FORTUS, D. School, teacher, peers, and parents' goals emphases and adolescents' motivation to learn science in and out of school. Journal of Research in Science Teaching, v. 50, n. 8, p. 952-988, 2019.

VERGARA, C; COFRÉ, H. Conocimiento Pedagógico del Contenido: ¿el paradigma perdido en la formación inicial y continua de profesores en Chile? Estudios pedagógicos (Valdivia), v. 40, n. Especial, p. 323-338, 2014.

WILSON, S. Professional development for science teachers. Science, v. 340, p. 310-313, 2013.

WILSON, S. Professional development for science teachers. Science, v. 340, p. 310-313, 2013.

YANG, X; GUO, X; YU, S. Student-generated content in college teaching: content quality, behavioural pattern and learning performance. Journal of Computer Assisted Learning, v. 32, n. 1, p. 1-15, 2016.

ZAPATA, M. Secuenciación de contenidos y objetos de aprendizaje. Revista de educación a Distancia, v. 5, n. 13, p. 1-14, 2005. 